\title{
Impedance of small obstacles and rough surfaces
}

\author{
G. V. Stupakov \\ Stanford Linear Accelerator Center, Stanford University, P.O. Box 4349, Stanford, California 94309
}

(Received 11 August 1998; published 27 October 1998)

\begin{abstract}
We calculate the longitudinal impedance for small obstacles of arbitrary shape located on the surface of a round perfectly conducting pipe. Calculations are carried out in a small-angle approximation that assumes a smallness of the angle between the surface of the obstacle and the unperturbed surface of the pipe. As an illustration of the accuracy of this approach, we compare the impedance of the triangular mask and an ellipsoidal protrusion in small-angle approximation with a more general result known from the literature. We also apply our theory to the calculation of the impedance due to the roughness of the wall surface in terms of the spectral function characterizing statistical properties of the microscopic surface landscape. [S1098-4402(98)00019-6]

PACS numbers: 41.75.-i, 41.20.-q
\end{abstract}

\section{INTRODUCTION}

Impedance calculation is an important part of accelerator physics that often helps one to understand beam dynamics and optimize the parameters of the machine. Trying to minimize the impedance, the design of new accelerators usually requires smoother surfaces and smaller obstacles in the vacuum chamber. Numerical calculations of the impedance for small obstacles are often difficult to perform because of the necessity of a fine mesh and high accuracy of calculations. On the other hand, using the smallness of the obstacle, one can try to develop a simplified perturbation theory that would give an analytical expression for the impedance.

The goal of this paper is to develop such a theory for small obstacles located on the surface of a round perfectly conducting pipe of an accelerator chamber. For simplicity, we limit our consideration by longitudinal impedance only, although transverse impedance can also be found using the same approach.

An important assumption that we will use in the derivation is a small-angle approximation. It means that the angle between the surface of the obstacle and the unperturbed surface of the pipe is almost everywhere small compared to unity. A similar approach has been used previously in a number of papers for periodic [1-3] and arbitrary [4] axisymmetric perturbations where the pipe radius $b$ was assumed to be perturbed as $b(z)=b_{0}+\epsilon s(z)$, with $\epsilon$ being a small formal parameter. Our result can be considered a generalization of the previous papers applicable for an arbitrary three-dimensional shape. It reduces to Warnock's result [4] in the axisymmetric case.

This requirement excludes from consideration sharp objects, but allows one to develop a general theory applicable for an arbitrary shape of the obstacle in a wide frequency range.

As the first step in the derivation, we find the amplitudes of the propagating waves radiated into the pipe when a beam passes by the obstacle. We then calculate the energy of the waves and relate the radiative energy loss of the beam to the real part of the impedance. Using Kramers-Kronig relation between the real and imaginary parts of the impedance, we find the total impedance for the obstacle.

As an illustration of the accuracy of the small-angle approximation, we compute the impedance for two different shapes whose impedance is known from the literature: a triangular mask [5] and a small ellipsoidal protrusion [6]. We show that our result for these shapes agrees in the limit of shallow shapes and has an addition numerical factor if the angles are not small.

As another practically important application of the theory, we derive the impedance of a perfectly conducting rough surface. As was recently pointed out in [7], roughness impedance can play an important role for short bunches (see, e.g., [8]). Our formula reduces calculation of the impedance for such surfaces to the integration of the spectral function characterizing statistical properties of the microscopic surface landscape.

\section{RADIATION FROM A SMOOTH OBSTACLE IN PIPE}

We consider a Fourier component of the relativistic beam propagating along the axes of the pipe with the current $I_{0} e^{-i \omega t+i \omega z / c}$. In a circular pipe of radius $b_{0}$, the beam carries the radial electric field

$$
\boldsymbol{E}^{b}(r, z)=\hat{\boldsymbol{r}} \frac{2 I_{0}}{c r} e^{i k z},
$$

where $k=\omega / c, c$ is the speed of light, and $\hat{\boldsymbol{r}}$ is the unit vector in the radial direction. In Eq. (1) and following, we drop the time dependent factor $e^{-i \omega t}$.

In a smooth pipe, the beam field is perpendicular to the wall surface and the electric field satisfies the boundary condition of zero tangential field for the perfectly conducting wall. With an obstacle, however, the electric field (1) will have a tangential component on the wall. We can easily find this component assuming that the shape of the 
obstacle is given by the equation

$$
r=b(z, \theta),
$$

where we use the cylindrical coordinates $r, \theta$, and $z$ with the axes $z$ directed along the pipe axis. Throughout this paper we will assume that the angle between the obstacle surface and that of the round pipe is small, which means

$$
|\nabla b| \ll 1 .
$$

This is the condition of small-angle approximation. We will also assume that the height of the obstacle is small compared to the pipe radius $b_{0}$,

$$
\left|b(\theta, z)-b_{0}\right| \ll b_{0} .
$$

In the small-angle approximation, the tangential component $\boldsymbol{E}_{t}$ of the beam electric field (1) on the surface of the obstacle is

$$
\boldsymbol{E}_{t}^{b}=\frac{2 I_{0}}{c b_{0}} e^{i k z}\left[0, \frac{1}{b_{0}} b_{\theta}(z, \theta), b_{z}(z, \theta)\right],
$$

where the three terms in the brackets denote radial, azimuthal, and axial components, respectively, and the subscript indicates the derivative with respect to the indicated variable.

The total electric field in the pipe with the obstacle can be represented as a sum of the vacuum beam field (1) and the radiation field $\boldsymbol{E}^{r}, \boldsymbol{E}=\boldsymbol{E}^{b}+\boldsymbol{E}^{r}$, where the latter satisfies the boundary condition

$$
\left.\boldsymbol{E}_{t}^{r}\right|_{\text {wall }}=-\left.\boldsymbol{E}_{t}^{b}\right|_{\text {wall }},
$$

so that the sum $\boldsymbol{E}^{r}+\boldsymbol{E}^{b}$ has a zero tangential component on the wall.

Because of the small-angle approximation, the tangential field $E_{t}^{b}$ is a small (first order) quantity proportional to the angle between the tangent to the surface and the pipe axis. Since we assume that the height of the obstacle is also small compared to the pipe radius [Eq. (4)], in order to find the radiation field in the first approximation, we can use the boundary condition (6) on the surface of the round pipe $r=b_{0}$ [rather than on the surface of the obstacle $r=b(r, z)]$. This introduces a second order error that is proportional to the product of $E_{t}^{b}$ and $b-b_{0}$, and can be neglected. Hence the electromagnetic problem reduces to solving the Maxwell equations in the circular pipe with a given nonzero tangential electric field on the wall. The solution can be found in textbooks on electrodynamics; below we will follow the formulation given in Ref. [9].

The radiated field far from the obstacle can be represented as a superposition of eigenmodes in the smooth pipe, $\mathrm{TE}_{n, m}$ and $\mathrm{TM}_{n, m}$,

$$
\boldsymbol{E}^{r}=\sum_{\mathrm{TE}, \mathrm{TM}} \sum_{n, m} a_{n, m}^{ \pm} \boldsymbol{E}_{n, m}^{ \pm}, \quad z \rightarrow \pm \infty,
$$

where we denote modes propagating in the positive and negative directions with the plus and minus signs, respectively. The amplitude of each mode $a_{n, m}^{ \pm}$can be found by integration of the tangential electric field $-\boldsymbol{E}_{t}^{b}$ over the wall surface [9],

$$
a_{n, m}^{ \pm}=-\frac{c}{4 \pi N_{n, m}} \int_{-\infty}^{\infty} d z \int_{0}^{2 \pi} d \theta \boldsymbol{H}_{n, m}^{\overline{+}} \cdot\left(\hat{\boldsymbol{r}} \times \boldsymbol{E}_{t}^{b}\right),
$$

where $\boldsymbol{H}_{n, m}$ is the magnetic field of the mode, and $N_{n, m}$ is the mode norm defined as

$$
\begin{aligned}
N_{n, m}= & -\frac{c}{4 \pi} \\
& \times \int r d r d \theta\left(\boldsymbol{E}_{n, m}^{+} \times \boldsymbol{H}_{n, m}^{-}+\boldsymbol{H}_{n, m}^{+} \times \boldsymbol{E}_{n, m}^{-}\right)_{z} .
\end{aligned}
$$

Having found the amplitudes of the radiated waves, we can calculate the energy $P$ lost by the beam per unit time as a result of the radiation. This energy is carried out to infinity by the propagating eigenmodes; hence

$$
P=\sum_{\mathrm{TE}, \mathrm{TM}} \sum_{n, m} P_{n, m}\left(\left|a_{n, m}^{+}\right|^{2}+\left|a_{n, m}^{-}\right|^{2}\right),
$$

where $P_{n, m}$ is the energy flow in the eigenmode of unit amplitude. The summation in Eq. (10) goes over the modes with the cutoff frequency below $\omega$.

Finally, we can relate the energy loss of the beam to the real part of the impedance [10]

$$
\operatorname{Re} Z(\omega)=\frac{2 P}{I_{0}^{2}} .
$$

\section{EIGENMODES}

In this section, we write down the expressions for the electric and magnetic fields in TM and TE modes in the straight circular pipe and calculate the energy flux and the norm of the modes.

The electric and magnetic fields in the $\mathrm{TM}_{n, m}$ mode of frequency $\omega$ are

$$
\begin{gathered}
\boldsymbol{E}_{n, m}=\left(\boldsymbol{\mathcal { E }}_{n, m}+\hat{z} E_{z n, m}\right) e^{i \sigma k_{n, m} z}, \\
\boldsymbol{H}_{n, m}=\mathcal{H}_{n, m} e^{i \sigma k_{n, m} z},
\end{gathered}
$$

where $\hat{z}$ is the unit vector in the $z$ direction, and

$$
\begin{gathered}
E_{z n, m}=\frac{\mu_{n, m}^{2}}{b_{0}^{2}} J_{n}\left(\mu_{n, m} \frac{r}{b_{0}}\right) e^{i n \theta}, \\
\mathcal{E}_{n, m}=\hat{\boldsymbol{r}} \frac{i \mu_{n, m} \sigma k_{n, m}}{b_{0}} J_{n}^{\prime}\left(\mu_{n, m} \frac{r}{b_{0}}\right) e^{i n \theta} \\
-\hat{\boldsymbol{\theta}} \frac{n \sigma k_{n, m}}{r} J_{n}\left(\mu_{n, m} \frac{r}{b_{0}}\right) e^{i n \theta}, \\
\mathcal{H}_{n, m}=\hat{\boldsymbol{r}} \frac{n \omega}{c r} J_{n}\left(\mu_{n, m} \frac{r}{b_{0}}\right) e^{i n \theta} \\
+\hat{\boldsymbol{\theta}} \frac{i \omega \mu_{n, m}}{c b_{0}} J_{n}^{\prime}\left(\mu_{n, m} \frac{r}{b_{0}}\right) e^{i n \theta},
\end{gathered}
$$


where $k_{n, m}=\sqrt{k^{2}-\left(\mu_{n, m} / b_{0}\right)^{2}}, J_{n}$ is the Bessel function of order $n$, and $\mu_{n, m}$ is the $m$ th root of $J_{n}$. The integer $n$ can take both positive and negative values, corresponding to the right and left circular polarizations of the waves. The variable $\sigma$ accounts for the direction of the propagation; it is equal to +1 for modes propagating along the $z$ axis and -1 for modes propagating in the opposite direction (the superscript in $\boldsymbol{E}^{ \pm}$and $\boldsymbol{H}^{ \pm}$in the previous section corresponds to $\sigma= \pm 1$ ). We define $k_{n, m}$ so that above the cutoff $k_{n, m}>0$; below the cutoff $k_{n, m}$ is purely imaginary with $\operatorname{Im} k_{n, m}>0$.

A simple integration gives the energy flow $P_{n, m}$ in a TM mode of unit amplitude

$$
P_{n, m}=\frac{1}{8} \omega k_{n, m} \mu_{n, m}^{2} J_{n}^{\prime 2}\left(\mu_{n, m}\right),
$$

and the norm of the mode

$$
N_{n, m}=-4 P_{n, m} \text {. }
$$

As we see, the norm of a TM mode is negative with the absolute value equal to four times the energy flow in the mode.

For TE modes we have

$$
\begin{gathered}
\boldsymbol{E}_{n, m}=\boldsymbol{E}_{n, m} e^{i k_{n, m} z}, \\
\boldsymbol{H}_{n, m}=\left(\mathcal{H}_{n, m}+\hat{z} H_{z n, m}\right) e^{i k_{n, m} z},
\end{gathered}
$$

where

$$
\begin{gathered}
H_{z n, m}=\frac{\nu_{n, m}^{2}}{b_{0}^{2}} J_{n}\left(\nu_{n, m} \frac{r}{b_{0}}\right) e^{i n \theta} \\
\mathcal{H}_{n, m}=\hat{\boldsymbol{r}} \frac{i \nu_{n, m} \sigma k_{n, m}}{b_{0}} J_{n}^{\prime}\left(\nu_{n, m} \frac{r}{b_{0}}\right) e^{i n \theta} \\
-\hat{\boldsymbol{\theta}} \frac{n \sigma k_{n, m}}{r} J_{n}\left(\nu_{n, m} \frac{r}{b_{0}}\right) e^{i n \theta}, \\
\mathcal{E}_{n, m}=-\hat{\boldsymbol{r}} \frac{n \omega}{c r} J_{n}\left(\nu_{n, m} \frac{r}{b_{0}}\right) e^{i n \theta} \\
-\hat{\boldsymbol{\theta}} \frac{i \omega \nu_{n, m}}{c b_{0}} J_{n}^{\prime}\left(\nu_{n, m} \frac{r}{b_{0}}\right) e^{i n \theta},
\end{gathered}
$$

$k_{n, m}=\sqrt{k^{2}-\left(\nu_{n, m} / b_{0}\right)^{2}}$, and $\nu_{n, m}$ is the $m$ th root of the derivative $J_{n}^{\prime}$. As above, $n$ varies from $-\infty$ to $\infty$, and $\sigma$ accounts for the direction of the propagation.

Calculating the energy flow in the mode, one finds

$$
P_{n, m}=\frac{1}{8} \omega k_{n, m} \nu_{n, m}^{2}\left(1-\frac{n^{2}}{\nu_{n, m}^{2}}\right) J_{n}^{2}\left(\nu_{n, m}\right),
$$

and the norm of the mode is equal to four times the energy flow

$$
\begin{aligned}
& N_{n, m}=4 P_{n, m} . \\
& Z_{\mathrm{TM}}(\omega)=\frac{2 k}{b_{0}^{2} c} \sum_{n, m} \frac{1}{k_{n, m}(\omega)} \int_{-\infty}^{\infty} \int_{-\infty}^{\infty} d z d u \tilde{s}_{n}^{\prime}(z)\left[\tilde{s}_{n}^{\prime}(u)\right]^{*} e^{i k(z-u)+i k_{n, m}|z-u|}, \\
& \text { and the result is }
\end{aligned}
$$

Note that, in contrast to the TM modes, the norm of the TE modes is positive.

\section{IMPEDANCE OF TM MODES}

We can now calculate the real part of the impedance for an obstacle of given shape given by Eq. (2). First, using Eq. (5), we find the tangential electric field on the surface of the obstacle and, using Eq. (8), we find the amplitudes of the radiated waves. With given amplitudes, using Eq. (10), we find the energy radiated by the beam and, using Eq. (11), we find the real part of the impedance. Since the radiated energy is a sum over all eigenmodes in the pipe, the total impedance $Z$ will be represented by contributions from TM and TE modes,

$$
Z=Z_{\mathrm{TM}}+Z_{\mathrm{TE}} .
$$

In this section, we will focus on the derivation of $Z_{\mathrm{TM}}$, leaving consideration of $Z_{\mathrm{TE}}$ for the following section.

The real part of the impedance for TM modes for positive real $\omega$ resulting from the calculations outlined above is given by the following expression:

$$
\operatorname{Re} Z_{\mathrm{TM}}(\omega)=\sum_{n=-\infty}^{\infty} \sum_{m=1}^{\infty} \sum_{\sigma= \pm 1} \operatorname{Re} Z_{n, m}(\omega, \sigma),
$$

where

$$
\begin{aligned}
& \operatorname{Re} Z_{n, m}(\omega, \sigma)=\frac{k}{b_{0}^{2} c k_{n, m}}\left|\hat{S}_{n}\left(k+\sigma k_{n, m}\right)\right|^{2}, \\
& k>\frac{\mu_{n, m}}{b_{0}}, \\
& \operatorname{Re} Z_{n, m}(\omega, \sigma)=0, \quad k<\frac{\mu_{n, m}}{b_{0}},
\end{aligned}
$$

and

$$
\begin{gathered}
\hat{S}_{n}(\xi)=\int_{-\infty}^{\infty} d z \tilde{s}_{n}^{\prime}(z) e^{i \xi z}, \\
\tilde{s}_{n}(z)=\frac{1}{2 \pi} \int_{0}^{2 \pi} d \theta \Delta b(z, \theta) e^{i n \theta},
\end{gathered}
$$

where $\Delta b=b(z, \theta)-b_{0}$ and the prime denotes the derivative with respect to the argument. We remind the reader here that for propagating TM modes $k_{m, n}=$ $\sqrt{k^{2}-\mu_{n, m}^{2} / b_{0}^{2}}>0$.

Using the Kramers-Kronig relation between the real part of the impedance and the full impedance (see, e.g., [10])

$$
Z(\omega)=-\frac{i}{\pi} \int_{-\infty}^{\infty} \frac{\operatorname{Re} Z\left(\omega^{\prime}\right) d \omega^{\prime}}{\omega^{\prime}-\omega},
$$

one can find the total impedance $Z$ by integration of $\operatorname{Re} Z(\omega)$. This calculation is performed in Appendix A, 
where the asterisk denotes complex conjugation.

Introducing the Fourier component $\hat{s}_{n}(\kappa)$

$$
\hat{s}_{n}(\kappa)=\frac{1}{2 \pi} \int_{-\infty}^{\infty} d k \tilde{s}_{n}(z) e^{i \kappa z},
$$

we obtain

$$
Z_{T M}(\omega)=\frac{2 k}{b_{0}^{2} c} \sum_{n, m} \frac{1}{k_{n, m}} \int_{-\infty}^{\infty} \int_{-\infty}^{\infty} \kappa \lambda \hat{s}_{n}(\kappa)\left[\hat{s}_{n}(\lambda)\right]^{*} d \kappa d \lambda \int_{-\infty}^{\infty} \int_{-\infty}^{\infty} d z d u e^{i k_{n, m}|z-u|-i(\lambda u-\kappa z)} .
$$

The internal integral over $z$ and $u$ can be easily computed if we assume, for convergence, that $k_{n, m}$ has a small positive imaginary part, due, e.g., to a weak damping of the modes,

$$
\int_{-\infty}^{\infty} \int_{-\infty}^{\infty} d z d u e^{i k_{n, m}|z-u|-i(\lambda u-\kappa z)}=-\frac{4 \pi i k_{n, m}}{(k+\lambda)^{2}-k_{n, m}^{2}} \delta(\kappa-\lambda),
$$

which reduces the impedance to

$$
Z_{\mathrm{TM}}(\omega)=-\frac{8 \pi i k}{c b_{0}^{2}} \sum_{n, m} \int_{-\infty}^{\infty} \lambda^{2} d \lambda \frac{\left|\hat{s}_{n}(\lambda)\right|^{2}}{(k+\lambda)^{2}-\left(k_{n, m}+i 0\right)^{2}},
$$

where we explicitly indicated that $k_{n, m}$ should be treated as having a small positive imaginary part. From this integral, we see that the real part of the impedance arises from the singular points of the integral where the denominator of the integrand vanishes.

In case of axisymmetric obstacle $r=b(z)$, all harmonics with $n \neq 0$ vanish, and only terms with $n=0$ contribute to Eqs. (32) and (36). In this limit, Eq. (32) reduces to the result obtained in Ref. [4].

\section{IMPEDANCE OF TE MODES}

Derivation of the impedance for TE modes $Z_{\mathrm{TE}}$ is analogous to the TM case. Below we will outline the calculations in this case.

The real part of the impedance for TE modes is given by

$$
\operatorname{Re} Z_{\mathrm{TE}}(\omega)=\sum_{n=-\infty}^{\infty} \sum_{m=1}^{\infty} \sum_{\sigma= \pm 1} \operatorname{Re} Z_{n, m}(\omega, \sigma)
$$

where now

$$
\begin{aligned}
& \operatorname{Re} Z_{n, m}(\omega, \sigma)=\frac{n^{2}}{b_{0}^{2} c k k_{n, m}\left(\nu_{n, m}^{2}-n^{2}\right)}\left|\hat{R}_{n, m}\left(k+\sigma k_{n, m}\right)\right|^{2}, \quad k>\frac{\nu_{n, m}}{b_{0}}, \\
& \operatorname{Re} Z_{n, m}(\omega, \sigma)=0, \quad k<\frac{\nu_{n, m}}{b_{0}},
\end{aligned}
$$

and

$$
\hat{R}_{n, m}(\xi)=\int_{-\infty}^{\infty} d z\left[\sigma k_{n, m} \tilde{s}_{n}^{\prime}(z)-i \frac{\nu_{n, m}^{2}}{b_{0}^{2}} \tilde{s}_{n}(z)\right] e^{i \xi z} .
$$

The total impedance in this case can be found from the Kramers-Kronig relation and, as shown in Appendix A, is given by

$$
Z(\omega)=\frac{\zeta(\omega)-\zeta(0)}{\omega}
$$

where

$$
\zeta(\omega)=\frac{2}{b_{0}^{6}} \sum_{n, m} \frac{n^{2}}{k_{n, m}\left(\nu_{n, m}^{2}-n^{2}\right)} \int_{-\infty}^{\infty} \int_{-\infty}^{\infty} d z d u \tilde{r}_{n, m}(z)\left[\tilde{r}_{n, m}(u)\right]^{*} e^{i k(z-u)+i k_{n, m}|z-u|},
$$

and

$$
\tilde{r}_{n, m}(z)=\frac{1}{2 \pi} \int_{0}^{2 \pi} d \theta\left[k_{n, m} b_{0}^{2} s_{z}(z, \theta)-i \nu_{n, m}^{2} s(z, \theta)\right] e^{i n \theta}
$$


Again, using Eq. (35) one can carry out the integration over $z$ and $u$ and, similar to Eq. (32), obtain

$$
Z_{\mathrm{TE}}(\omega)=-\frac{8 k \pi i}{c b_{0}^{2}} \sum_{n, m} \frac{n^{2}}{\left(\nu_{n, m}^{2}-n^{2}\right)} \int_{-\infty}^{\infty} d \lambda \frac{\lambda^{2}\left|\hat{s}_{n}(\lambda)\right|^{2}}{\left(k+\lambda^{2}\right)-\left(k_{n, m}+i 0\right)^{2}} .
$$

For the axisymmetric case, when $\hat{s}_{n}=0$ for $n \neq 0$, the impedance of TE modes vanishes.

\section{TOTAL IMPEDANCE FOR SMALL OBSTACLES}

Our result given by Eqs. (32) and (43) is valid for arbitrary frequency and transverse size (width and length) of the obstacle under the conditions given by Eqs. (3) and (4). For example, these equations can be applied for smooth transitions of the length much larger than the chamber radius. In this paper, however, we are mainly interested in the limit when the characteristic transverse size of the obstacle, which we denote by $g$, is much smaller than the pipe radius $b_{0}$. We will also assume that the frequency of interest is not very large compared with the inverse transverse size of the obstacle multiplied by the speed of light, so that $k g \ll 1^{1}$. In this limit, as we will show below, the expression for the impedance can be significantly simplified.

It turns out that in the limit $g \ll b_{0}$, the main contribution to the sums in Eqs. (32) and (43) comes from the terms such that $\left|k_{n, m}\right| \sim 1 / g$, and hence $n, m \gg 1$. This means that $\nu_{n, m} \approx \mu_{n, m}$ and $k_{n, m} \approx i \mu_{n, m} / b_{0}$, and we can neglect $k$ in comparison with $\lambda$ in Eqs. (32) and (43),

$$
\begin{aligned}
Z(\omega)= & Z_{\mathrm{TM}}(\omega)+Z_{\mathrm{TE}}(\omega) \\
\approx & -\frac{8 k \pi i}{c b_{0}^{2}} \sum_{n, m}\left[\frac{n^{2}}{\left(\nu_{n, m}^{2}-n^{2}\right)}+1\right] \\
& \times \int_{-\infty}^{\infty} d \lambda \frac{\lambda^{2}\left|\hat{s}_{n}(\lambda)\right|^{2}}{\lambda^{2}+\left(\nu_{n, m} / b_{0}\right)^{2}} .
\end{aligned}
$$

The summation over index $m$ can be carried out, and, as shown in Appendix B, one can obtain the following result:

$$
Z(\omega)=-\frac{4 k \pi i}{c b_{0}^{2}} \int_{-\infty}^{\infty} d \lambda \int_{-\infty}^{\infty} d n\left|\hat{s}_{n}(\lambda)\right|^{2} \frac{\lambda^{2} b_{0}^{2}}{\sqrt{b_{0}^{2} \lambda^{2}+n^{2}}} .
$$

It is convenient to introduce here new variables $\kappa_{z}=$ $\lambda, \kappa_{x}=n / b_{0}$, and $\kappa=\sqrt{\kappa_{z}^{2}+\kappa_{x}^{2}}$, so that

$$
Z(\omega)=-\frac{4 i k \pi}{c b_{0}^{2}} \int_{-\infty}^{\infty} d \kappa_{z} \int_{-\infty}^{\infty} d \kappa_{x}\left|\hat{s}\left(\kappa_{z}, \kappa_{x}\right)\right|^{2} \frac{\kappa_{z}^{2}}{\kappa}
$$

where we use the notation $\hat{s}\left(\kappa_{z}, \kappa_{x}\right)=b_{0} \hat{s}_{n}(\lambda)$. The variables $\kappa_{z}$ and $\kappa_{x}$ have a meaning of wave numbers in $z$ and $x$ directions, respectively, in a local coordinate

\footnotetext{
${ }^{1}$ Note that this frequency can be much larger than the cutoff frequency $\sim c / b_{0}$.
}

system related to the tangential plane at the location of the obstacle, with $x=b_{0} \theta$ (see Fig. 1). Recalling the definitions of Eqs. (30) and (33) one can write

$$
\hat{s}\left(\kappa_{z}, \kappa_{x}\right)=\frac{1}{4 \pi^{2}} \int_{-\infty}^{\infty} d z d x \Delta b(x, z) e^{i \kappa_{x} x+i \kappa_{z} z},
$$

where $\hat{s}\left(\kappa_{z}, \kappa_{x}\right)$ is now expressed in terms of the bump shape in the local coordinate system on the surface of the wall.

For an axisymmetric protrusion $r=b(z)$, one can show that in place of Eq. (46) one obtains

$$
Z(\omega)=-\frac{4 i k \pi}{c b_{0}} \int_{-\infty}^{\infty} d \kappa_{z}\left|\hat{s}\left(\kappa_{z}\right)\right|^{2}\left|\kappa_{z}\right|
$$

where

$$
\hat{s}\left(\kappa_{z}\right)=\frac{1}{2 \pi} \int_{-\infty}^{\infty} d z \Delta b(z) e^{i \kappa_{z} z}
$$

\section{TWO EXAMPLES}

In this section, we calculate the impedance of an ellipsoidal bump and a triangular mask using expressions from the previous section and compare them with results known from the literature.

First, consider a small ellipsoidal protrusion in the chamber, for which

$$
\Delta b(z, \theta) \equiv-y=-h_{0} \sqrt{g^{2}-x^{2}-z^{2}},
$$

where $h_{0}$ is the height and $g$ is the width of the ellipsoid (see Fig 2). Assuming that $g \ll b_{0}$, in the limit

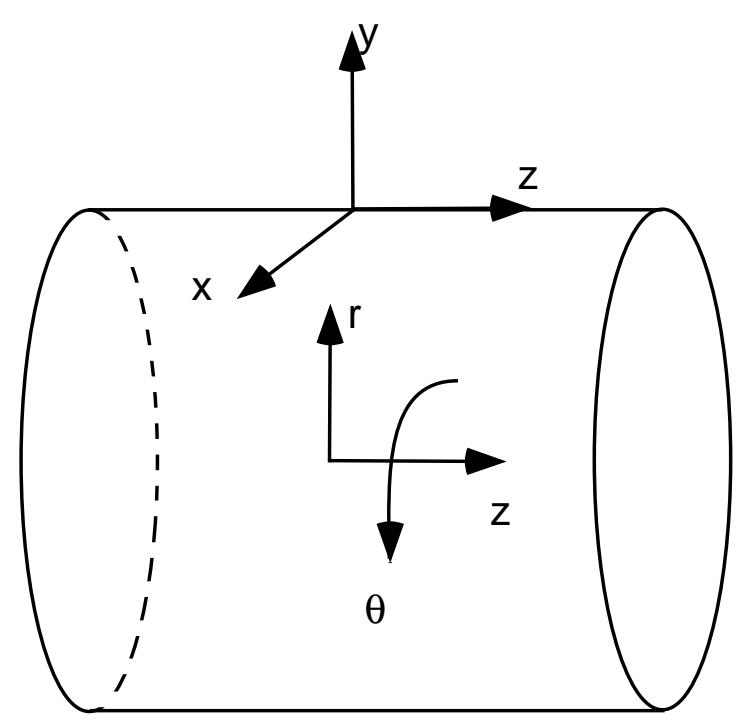

FIG. 1. A local Cartesian coordinate system $x y z$ on the surface and a cylindrical coordinate system $r \theta z$ in the vacuum chamber of circular cross section. 


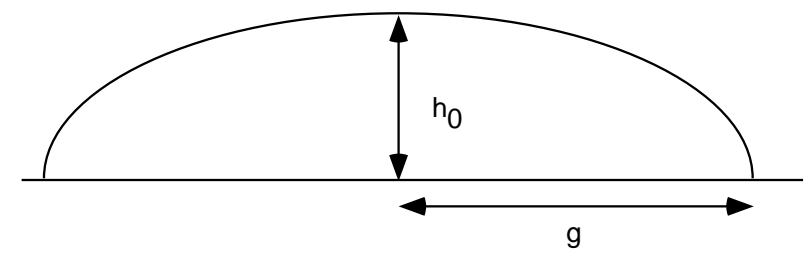

FIG. 2. Ellipsoidal protrusion on the wall.

of frequencies such that $g k \ll 1$, the impedance of such an obstacle in small-angle approximation can be found using Eq. (46). Note that the small-angle approximation requires that $h_{0} \ll g$; this condition guarantees that the angle between the bump surface and the horizontal plane in Fig. 2 is small everywhere except the edges where this angle equals $90^{\circ}$.

The Fourier image (47) for the ellipsoid (50) can be easily found

$$
\hat{s}(\kappa)=\frac{h_{0} g^{2}}{2 \pi} \frac{\sin \kappa-\kappa \cos \kappa}{\kappa^{3}},
$$

which gives the following result for the impedance of the ellipsoid:

$$
\begin{aligned}
Z_{s a} & =-i \frac{Z_{0} k h^{2} g}{4 \pi b_{0}^{2}} \int_{0}^{\infty} d \kappa \frac{\sin \kappa-\kappa \cos \kappa}{\kappa^{4}} \\
& =-i \frac{Z_{0} k h_{0}^{2} g}{24 b_{0}^{2}}
\end{aligned}
$$

where the subscript $s a$ indicates that this formula is obtained in the small-angle approximation.

This result can be compared with a more general expression obtained in Ref. [6] and valid, in the limit of small frequencies, for an arbitrary ratio of $h_{0}$ and $g$ (but $\left.g, h_{0} \ll b_{0}\right)$,

$$
Z=-i \frac{Z_{0} k h_{0} g^{2}}{6 \pi b_{0}^{2}}\left\{I_{1}^{-1}\left(\frac{h_{0}}{g}\right)+\left[I_{2}\left(\frac{h_{0}}{g}\right)-1\right]^{-1}\right\},
$$

where

$$
I_{n}(x)=\frac{x}{2} \int_{0}^{\infty} \frac{d \xi}{(\xi+1)^{n}\left(\xi+x^{2}\right)^{\frac{5}{2}-n}} .
$$

The ratio $Z_{s a} / Z$ is shown in Fig. 3. It is seen that, for small values of $h_{0} / g, Z_{s a}$ agrees with the exact formula (53); however, for larger aspect ratios, the smallangle approximation underestimates the impedance. For $h_{0} / g=1$, corresponding to a semisphere, the small-angle approximation gives about 2 times smaller result. We remind the reader here that the assumption of the smallangle approximation breaks down when $h_{0} \sim g$.

Another example where the small-angle approximation can be compared with a more accurate theory is the case of the axisymmetric triangular mask shown in Fig. 4. Using Eq. (49) for the Fourier spectrum of the triangular

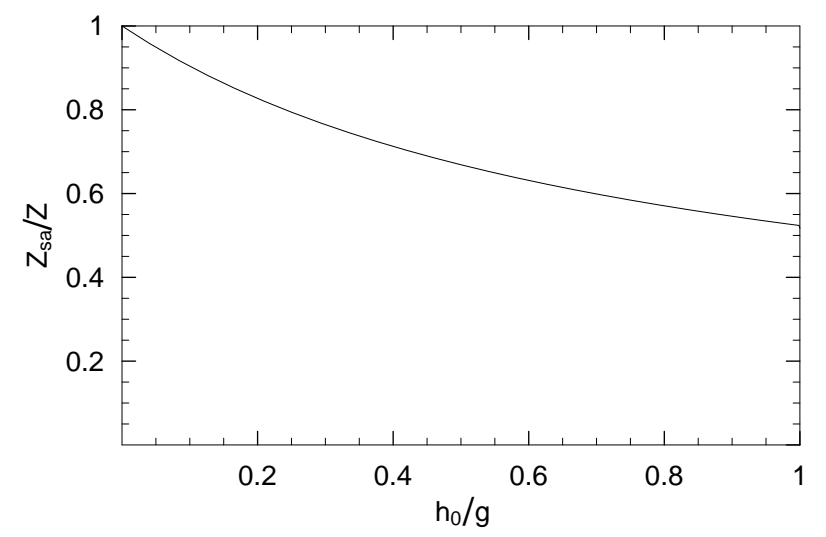

FIG. 3. Ratio of the small-angle approximation impedance $Z_{\text {sa }}$, Eq. (52), and $Z$ given by Eq. (53) as a function of the ellipsoid aspect ratio.

shape, one finds

$$
\hat{s}(\kappa)=-\frac{2 h_{0}}{\pi g \kappa^{2}}\left(\cos \frac{g \kappa}{2}-1\right) .
$$

Putting this into Eq. (48) gives for impedance in the small-angle approximation $\left(h_{0} \ll g\right)$

$$
Z_{s a}=-2(\ln 2) \frac{i k Z_{0} h_{0}^{2}}{\pi^{2} b_{0}} .
$$

A more general formula for the triangular mask, valid even for large ratios $h_{0} / g$ (but $h_{0}, g \ll b_{0}$ ), was derived in Ref. [5],

$$
Z=-\frac{i k Z_{0}}{4 \pi b_{0}}\left(\alpha_{e}+g h_{0}\right)
$$

where

$$
\alpha_{e}=-2 \nu\left[\frac{\pi h_{0}}{\sin (\pi \nu) \Gamma(1 / 2-\nu) \Gamma(1+\nu)}\right]^{2},
$$

and $\nu$ is defined by

$$
\tan \pi \nu=\frac{2 h_{0}}{g}
$$

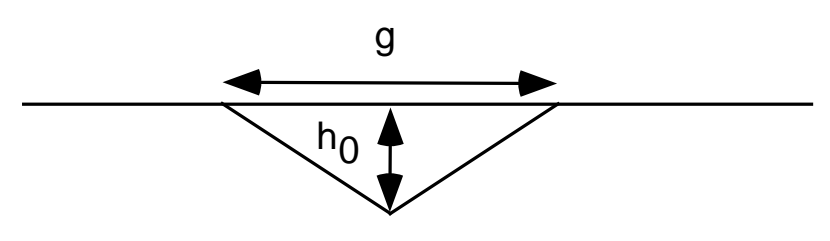

FIG. 4. Axisymmetric triangular mask. The dashed line shows the axis of the pipe. 


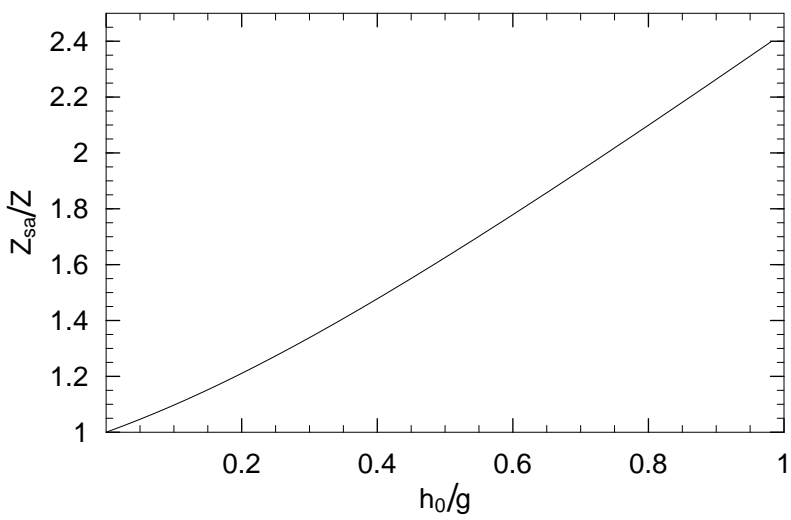

FIG. 5. Ratio of the small-angle approximation impedance $Z_{s a}$, Eq. (56), and $Z$ given by Eq. (57) as a function of the triangle aspect ratio.

A comparison of the two results is shown in Fig. 5. Again, we have a good agreement between the two models in the limit $h_{0} \ll g$; however, increasing $h_{0} / g$ beyond the limit of the applicability of the small-angle approximation leads to the overestimation of the impedance by $Z_{s a}$.

\section{RANDOM SURFACE}

With a small modification, our analysis can also be applied to the case of a rough surface. We assume that such a surface consists of randomly distributed bumps with a characteristic scale of the bump $g \ll b_{0}$, and the frequency of interest $\omega$ satisfies the inequality $\omega g / c \ll$ 1. The small-angle approximation also assumes that the typical angle between the tangent to the surface and the horizontal plane is small. We can use a statistical description of the rough surface in terms of the correlation function and its spectrum. In this approach, the impedance given by Eq. (46) is averaged over the random distribution of surface bumps, and the averaged value $\left\langle\left|\hat{s}\left(\kappa_{z}, \kappa_{x}\right)\right|^{2}\right\rangle$ is used in Eq. (46) instead of $\left|\hat{s}\left(\kappa_{z}, \kappa_{x}\right)\right|^{2}$. To carry out the averaging we start from the equation

$$
\begin{aligned}
\left|\hat{s}\left(\kappa_{z}, \kappa_{x}\right)\right|^{2}= & \frac{1}{(2 \pi)^{4}} \int d x d y \Delta b(x, z) e^{i \kappa_{z} z+i \kappa_{x} x} \\
& \times \int d x^{\prime} d y^{\prime} \Delta b\left(x^{\prime}, z^{\prime}\right) e^{-i \kappa_{z} z^{\prime}-i \kappa_{x} x^{\prime}},
\end{aligned}
$$

which directly follows from the definition of $\hat{s}\left(\kappa_{z}, \kappa_{x}\right)$. Averaging this equation gives

$$
\begin{aligned}
\left\langle\left|\hat{s}\left(\kappa_{z}, \kappa_{x}\right)\right|^{2}\right\rangle= & \frac{1}{(2 \pi)^{4}} \int d x d y d x^{\prime} d y^{\prime} \\
& \times\left\langle\Delta b\left(x^{\prime}, z^{\prime}\right) \Delta b(x, z)\right\rangle e^{i \kappa_{z}\left(z-z^{\prime}\right)+i \kappa_{x}\left(x-x^{\prime}\right)} .
\end{aligned}
$$

At this point we introduce the correlation function $K(x, z)$ such that

$$
K\left(x-x^{\prime}, z-z^{\prime}\right)=\left\langle\Delta b\left(x^{\prime}, z^{\prime}\right) \Delta b(x, z)\right\rangle,
$$

and the spectrum $R\left(\kappa_{z}, \kappa_{x}\right)$, equal to the Fourier image of the correlation function,

$$
R\left(\kappa_{z}, \kappa_{x}\right)=\frac{1}{(2 \pi)^{2}} \int d x d z K(x, z) e^{-i \kappa_{z} z-i \kappa_{x} x} .
$$

Putting Eqs. (62) and (63) into Eq. (61) and performing integration over the surface of a pipe of length $L$ gives

$$
\left\langle\left|\hat{s}\left(\kappa_{z}, \kappa_{x}\right)\right|^{2}\right\rangle=\frac{b_{0} L}{2 \pi} R\left(\kappa_{z}, \kappa_{x}\right),
$$

with the averaged impedance of the rough surface

$$
Z(\omega)=-\frac{i k Z_{0} L}{2 \pi b_{0}} \int d \kappa_{z} d \kappa_{x} R\left(\kappa_{z}, \kappa_{x}\right) \frac{\kappa_{z}^{2}}{\kappa} .
$$

For an isotropic surface, such that all directions on the surface are statistically equivalent, the function $R$ depends only on the absolute value of the vector $\left(\kappa_{x}, \kappa_{z}\right)$, $R=R(\kappa)$, and Eq. (65) reduces to

$$
Z(\omega)=-\frac{i k Z_{0} L}{2 b_{0}} \int_{0}^{\infty} \kappa^{2} d \kappa R(\kappa) .
$$

As an example of statistical description of the surface, we consider here a model of a fractal landscape, with a spectral function decaying as a power of the absolute value of the vector $\kappa$,

$$
\begin{aligned}
& R(\kappa)=\frac{A}{\kappa^{q}}, \quad \kappa>\kappa_{0}, \\
& R(\kappa)=0, \quad \kappa<\kappa_{0} .
\end{aligned}
$$

A picture of such a surface is shown in Fig. 6. In order to avoid divergence at small values of $\kappa$, we limited the spectrum from below by some small value $\kappa_{0}$. The constant $A$ is related to the rms height $d$ of the surface bumps

$$
d^{2}=2 \pi \int_{0}^{\infty} \kappa d \kappa R(\kappa)=\frac{2 \pi A}{q-2} \kappa_{0}^{2-q},
$$

where we assume $q>2$ for convergence of the integral.

Calculating the impedance with Eq. (66), we find the impedance of a round pipe whose internal surface is characterized by the rms height of the bumps $d$ and parameter $\kappa_{0}$

$$
Z(\omega)=-\frac{i k Z_{0}}{4 \pi b_{0}} \frac{q-2}{q-3} d^{2} \kappa_{0} .
$$

The inverse parameter $\kappa_{0}^{-1}$ can be associated with a characteristic correlation length of the bumps on the surface $l_{\text {corr }} \sim \kappa_{0}^{-1}$. The structures on the surface tend to be uncorrelated on the distance that is much larger than $l_{\text {corr. }}$. Equation (69) shows that the impedance not only depends on the rms height of the bumps, but also on the correlation between the location of the bumps relative to each other. 

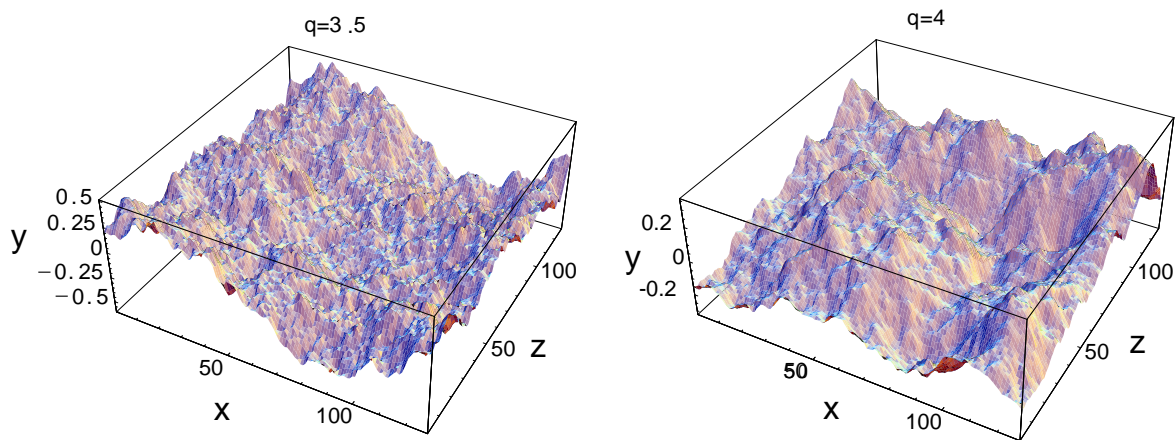

FIG. 6. (Color) Fractal surfaces for $q=3.5$ and $q=4$ (in arbitrary units). Smaller values of $q$ give more "spiky" profiles.

\section{CONCLUSION}

In this paper, we derived a general expression for the longitudinal impedance, Eqs. (36) and (43), of a protrusion of arbitrary shape located on the surface of a perfectly conducting cylindrical pipe. We assumed that the height of the protrusion is much smaller than the pipe radius, and we also used a small-angle approximation for the shape of the protrusion. These general expressions for the impedance can be significantly simplified in the limit of relatively small frequencies $\omega g / c \ll 1$, with the result given by Eq. (46). The impedance in this limit is purely inductive and can be easily computed for an arbitrary shape of the protrusion. In two examples, we showed that our result agrees with previously calculated impedance for these specific shapes. Those examples also demonstrate the accuracy of the small-angle approximation-even when this approximation is formally not valid, it still gives, within a numerical factor, the right expression for the impedance.

Extending our consideration, we applied it to the case of a rough surface. For such a surface, the result can be expressed in terms of the spectrum of the roughness. In a simple example of a fractal landscape, we showed how to calculate the impedance. Our result indicates that not only rms height of the surface is important, but correlation properties of the landscape should also be taken into consideration.

\section{ACKNOWLEDGMENTS}

The author is thankful to K. Bane, M. Cornacchia, and P. Emma for useful discussions. This work was supported by Department of Energy Contract No. DEAC03-76SF00515.

\section{APPENDIX A}

To calculate the total impedance of TM modes, we will represent it as a sum of contributions from different eigenmodes $n, m$ [see Eqs. (27) and (31)],

$$
Z_{\mathrm{TM}}(\omega)=\sum_{n=-\infty}^{\infty} \sum_{m=1}^{\infty} Z_{n, m}(\omega)
$$

where

$$
Z_{n, m}(\omega)=-\frac{i}{\pi} \sum_{\sigma= \pm 1} \int_{-\infty}^{\infty} \frac{\operatorname{Re} Z_{n, m}\left(\omega^{\prime}, \sigma\right) d \omega^{\prime}}{\omega^{\prime}-\omega},
$$

and $\operatorname{Re} Z_{n, m}$ is given by Eq. (28). In the last integral, in order to avoid singularity at $\omega=\omega^{\prime}$, one has to assume that $\omega$ has a small positive imaginary part, as shown in Fig. 1. Since $\operatorname{Re} Z_{n, m}$ is zero below the cutoff frequency, the integration path in the complex plane $\omega^{\prime}$ goes from the cutoff frequency $c \mu_{n, m} / b_{0}$ to $\infty$ along the real axis and from $-\infty$ to $-c \mu_{n, m} / b_{0}$, as shown in Fig. 7. (Note that the real part of the impedance is an even function of frequency for real $\omega^{\prime}$.)

We now define the longitudinal wave number $k_{n, m}\left(\omega^{\prime}\right)=\left(\omega^{\prime 2} / c^{2}-\mu_{n, m}^{2} / b_{0}^{2}\right)^{1 / 2}$ as a function of the complex variable $\omega^{\prime}$, so that it is an analytic function in the complex plane with two cuts going along the real axis as shown in Fig. 8. As shown in this figure, $k_{n, m}\left(\omega^{\prime}\right)$ is real on the edges of the cuts and takes positive and negative signs there. With this definition, we can now change the integration path of Fig. 7 to two contours $C_{1}$ and $C_{2}$, shown in Fig. 9, that go on the upper and lower edges of the cuts passing around the cutoff points,

$$
Z_{n, m}(\omega)=-\frac{i}{\pi} \int_{C_{1}+C_{2}} \frac{\operatorname{Re} Z_{n, m}\left(\omega^{\prime}, \sigma\right) d \omega^{\prime}}{\omega^{\prime}-\omega} .
$$

Note that the summation over $\sigma$ is removed in Eq. (A3) because the contributions from negative values of $\sigma$ are now included in the lower branches of the contours $C_{1}$ and $C_{2}$. It turns out that the integral in Eq. (A3) does not

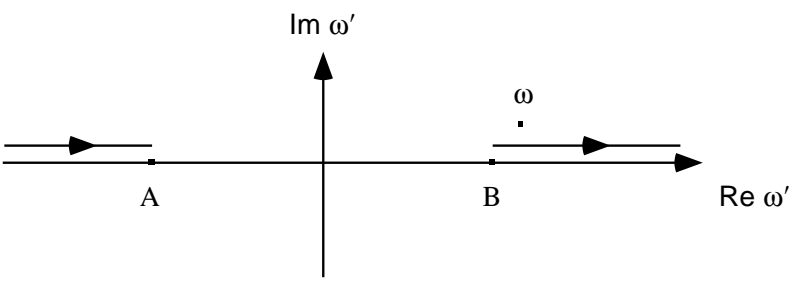

FIG. 7. Complex plane $\omega^{\prime}$ and the integration path for Eq. (A2). The points A and B correspond to the cutoff frequency $-c \mu_{n, m} / b_{0}$ and $c \mu_{n, m} / b_{0}$, respectively. The frequency $\omega$ has an infinitesimally small positive imaginary part. 


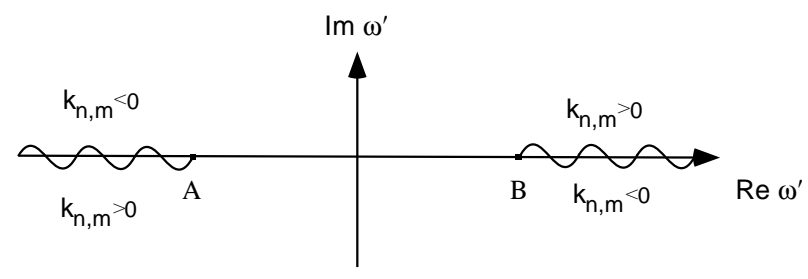

FIG. 8. Complex plane $\omega^{\prime}$ with cuts indicated by the wavy line. The first cut goes along the real axis from $-\infty$ to $\mathrm{A}$, and the second one from B to $\infty$. Function $k_{n, m}\left(\omega^{\prime}\right)$ takes positive and negative values on the edges of the cuts, as shown in the figure.

depend on $\sigma$, and both positive and negative values of $\sigma$ on the right-hand side give the same result.

Using Eq. (29) we get

$$
\begin{aligned}
\left|\hat{S}_{n}\left(k+\sigma k_{n, m}\right)\right|^{2}= & \int_{-\infty}^{\infty} \int_{-\infty}^{\infty} d z d u \tilde{s}_{n}^{\prime}(z)\left[\tilde{s}_{n}^{\prime}(z)\right]^{*} \\
& \times e^{i\left(k+\sigma k_{n, m}\right)(z-u)}
\end{aligned}
$$

and

$$
\begin{aligned}
Z_{n, m}(\omega)= & -\frac{i}{\pi b_{0}^{2} c^{2}} \int_{-\infty}^{\infty} \int_{-\infty}^{\infty} d z d u \tilde{s}_{n}^{\prime}(z)\left[\tilde{s}_{n}^{\prime}(z)\right]^{*} \\
& \times \int_{C_{1}+C_{2}} \frac{d \omega^{\prime}}{\omega^{\prime}-\omega} \frac{\omega^{\prime}}{k_{n, m}\left(\omega^{\prime}\right)} e^{i\left(k+\sigma k_{n, m}\right)(z-u)} .
\end{aligned}
$$

We can now close the integration path by infinitely large semicircles as shown in Fig. 9. The contribution to the integral from those semicircles will vanish because of the exponential factor $e^{-\sigma \operatorname{Im} k_{m, n}(z-u)}$ in the integrand, if we choose $\sigma=1$ for $z-u>0$ and $\sigma=-1$ for $z-u<0$. Then the integral reduces to the residue at $\omega^{\prime}=\omega$, and we obtain as a result Eq. (32).

Calculations for TE modes are completely analogous to the TM case with the same transformation of the integration path from the one shown in Fig. 7 to the closed

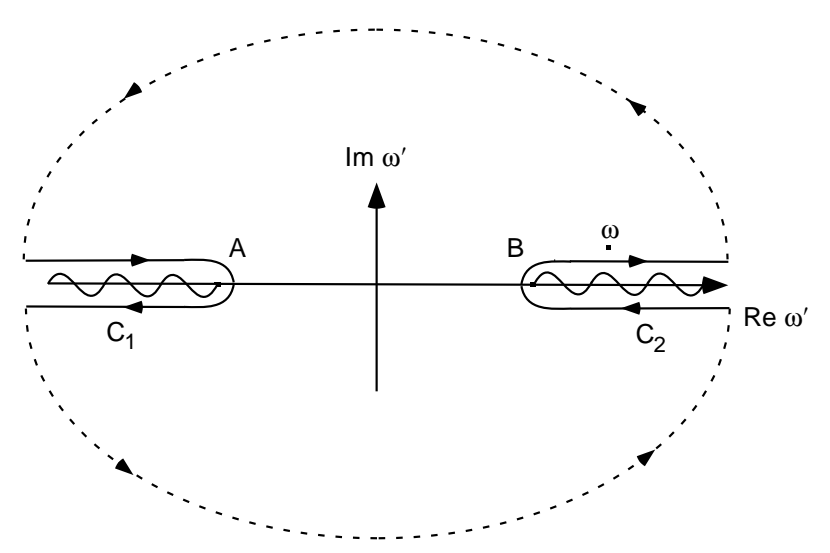

FIG. 9. Complex plane $\omega^{\prime}$ with the integration contours $C_{1}$ and $C_{2}$. The path $C_{1}+C_{2}$ can be closed by adding infinitely large semicircles shown by dashed lines.

loop in Fig. 9 (with the cutoff frequency now $\pm c \nu_{n, m} / b_{0}$ rather than $\left.\pm c \mu_{n, m} / b_{0}\right)$. The only difference arises from the fact that the real part of the impedance, Eq. (38), has a singularity at $\omega=0$. As a result, after closing the contour of the integration, there will be two residues in the integral: one at $\omega^{\prime}=\omega$ and the other at $\omega^{\prime}=0$. This leads to the expression Eq. (40) in which the first term in the numerator is due to the residue at $\omega$, and the second one is due to the residue at the origin.

\section{APPENDIX B}

For large values of $n$ and $m$, one can use the following identity for the roots of the Bessel functions [11]

$$
\nu_{m, n} \approx n f\left(\frac{m}{n}\right),
$$

where the function $f(x)$ is defined implicitly by the equation

$$
x f(x)=\sqrt{f^{2}-1}-\frac{1}{f} .
$$

Putting Eq. (B1) into Eq. (44) gives

$$
\begin{aligned}
Z(\omega) & =-\frac{8 k \pi i}{c b_{0}^{2}} \sum_{n, m} \frac{f^{2}}{f^{2}-1} \int d \lambda \frac{\lambda^{2}\left|\hat{s}_{n}(\lambda)\right|^{2}}{\lambda^{2}+\left(n f / b_{0}\right)^{2}} \\
& \approx-\frac{8 k \pi i}{c b_{0}^{2}} \int d m d n \frac{f^{2}}{f^{2}-1} \int d \lambda \frac{\lambda^{2}\left|\hat{s}_{n}(\lambda)\right|^{2}}{\lambda^{2}+\left(n f / b_{0}\right)^{2}},
\end{aligned}
$$

where we used integration over $n$ and $m$ instead of summation, which is valid because the main contribution to the sum comes from large $n$ and $m$. Changing the integration variable from $m$ to $f$, so that $d m=$ $\sqrt{f^{2}-1} d f / f$, and using the identity

$$
\int_{1}^{\infty} \frac{f d f}{\sqrt{f^{2}-1}\left(f^{2}+a^{2}\right)}=\frac{\pi}{2 \sqrt{1+a^{2}}},
$$

one obtains Eq. (46).
[1] M. Chatard-Moulin and A. Papiernik, IEEE Trans. Nucl. Science NS-26, 3523 (1979).

[2] S. Krinsky, in Proceedings of the XIth Particle Accelerator Conference, Geneva, Switzerland, 1980 (Birkhäuser Verlag, Basel, Switzerland, 1980), p. 576.

[3] R. K. Cooper, S. Krinsky, and P.L. Morton, Part. Accel. 12, 1 (1982).

[4] R.L. Warnock, SLAC Report No. SLAC-PUB-6038 (unpublished).

[5] S. S. Kurennoy and G. V. Stupakov, Part. Accel. 45, 95 (1994). 
[6] S. S. Kurennoy, Phys. Rev. E 55, 3529 (1997).

[7] K.L.F. Bane, C. K. Ng, and A. W. Chao, SLAC Report SLAC-PUB-7514 (unpublished).

[8] SLAC Report SLAC-R-521 (unpublished).

[9] L. A. Weinstein, Electromagnetic Waves (Radio i svyaz',
Moscow, 1988), in Russian.

[10] A. W. Chao, Physics of Collective Beam Instabilities in High Energy Accelerators (Wiley, New York, 1993).

[11] M. Abramowitz and I. A. Stegun, Handbook of Mathematical Functions (Dover, New York, 1968). 